\title{
Use of olanzapine compared with clozapine for treatment-resistant schizophrenia in a real-world setting: nationwide register-based study
}

\author{
Young Tak Jo, Sung Woo Joo, Soojin Ahn, Youngjae Choi and Jungsun Lee
}

\section{Background}

Clozapine is generally considered as the treatment of choice for patients with treatment-resistant schizophrenia (TRS). However, its superiority has recently been questioned because olanzapine has been suggested as non-inferior to clozapine in its effectiveness.

\section{Aims}

We aimed to investigate the current status of clozapine prescriptions to identify any disparity between clinical guidelines and real-world practices.

\section{Method}

In this study, we utilised the Health Insurance Review Agency database in the Republic of Korea to investigate the real-world effectiveness of clozapine for patients with TRS. We compared differences in patient variables before and after clozapine administration, and we also performed survival analyses for both psychiatric admissions and emergency room visits among patients who used clozapine or olanzapine.

\section{Results}

This study investigated an incident cohort of 64442 patients, and 2338 patients have been prescribed clozapine. Of these, 998 patients had TRS. In survival analysis, clozapine showed a worse survival rate for psychiatric admissions than olanzapine (hazard ratio 0.615$)$. We also identified that clinicians tended to try a number of antipsychotics, as recommended, before starting patients on clozapine.

\section{Conclusions}

In conclusion, we found that olanzapine led to higher survival rates for psychiatric admissions than clozapine. Thus, considering the risk of serious adverse effects, clozapine may be used conservatively. Considering several studies advocating superior efficacy of clozapine, further studies with extensive data are recommended.

\section{Keywords}

Schizophrenia; clozapine; olanzapine; national health programs; delivery of healthcare.

\section{Copyright and usage}

(C) The Author(s), 2021. Published by Cambridge University Press on behalf of the Royal College of Psychiatrists. This is an Open Access article, distributed under the terms of the Creative Commons Attribution licence (http://creativecommons.org/ licenses/by/4.0/), which permits unrestricted re-use, distribution, and reproduction in any medium, provided the original work is properly cited.

\section{Background}

Schizophrenia is a chronic and debilitating disorder characterised by hallucinations, delusions and disorganised thoughts. As schizophrenia reduces the mental function of patients and often seriously impairs their quality of life, aggressive treatment is often considered. However, approximately $30 \%$ of patients with schizophrenia cannot achieve remission, even with appropriate antipsychotics use. ${ }^{1}$ This is referred to as treatment-resistant schizophrenia (TRS). ${ }^{2}$ According to the available clinical guidelines, ${ }^{3,4}$ clozapine is generally considered as the treatment of choice for patients with TRS.

\section{Clozapine}

Clozapine, which was first sold commercially in 1972 as the first atypical antipsychotic, has been known as the most effective antipsychotic. despite some adverse effects including fatal agranulocytosis. ${ }^{5}$ It has been widely prescribed because of its higher efficacy compared with other atypical antipsychotics. Several studies have demonstrated the efficacy and cost-effectiveness of clozapine for patients with TRS. ${ }^{6-9}$

\section{Olanzapine}

However, the superiority of clozapine has recently been questioned. Some studies have suggested that the superiority of clozapine compared with olanzapine in terms of effectiveness is uncertain. ${ }^{10-13}$ In other words, olanzapine is non-inferior to clozapine. Olanzapine has been considered superior to other atypical antipsychotics. ${ }^{14}$ Based on this perspective, considering the risk of serious adverse effects ${ }^{15}$ and the cost of routine laboratory check-ups, more conservative usage of clozapine might be considered.

\section{Aims}

A comprehensive study using real-world data is therefore warranted. It should be clarified whether or not clozapine should be used as the treatment of choice for patients with TRS. It has been suggested that realworld practice relating to antipsychotics may be different from welldesigned study settings. ${ }^{16}$ Although a few studies have investigated real-world data for clozapine use, ${ }^{16-18}$ this has been limited considering the widespread use of clozapine. It has been challenging to investigate real-world data for patients who use clozapine because most countries do not have a nationwide healthcare register for such research.

In this study, we utilised the Korean nationwide healthcare register to investigate the real-world effectiveness of clozapine for patients with TRS. Specifically, we investigated data from the Health Insurance Review \& Assessment Service (HIRA) database between 1 January 2007 and 31 December 2016, to determine the real-world effectiveness of clozapine for patients with TRS, especially compared with olanzapine, and we also identified additional information including the prevalence of use and prescribing patterns.

\section{Method}

\section{Nationwide register (HIRA)}

In this study, we utilised the data from the HIRA database. ${ }^{19}$ In the Republic of Korea, there is a single government-payer health 
coverage system, the National Health Insurance Service, that covers approximately $98 \%$ of the national population. The HIRA can be used to review medical fees and the attributes of healthcare. For this purpose, HIRA has constructed and maintained a vast nationwide database comprising all medical claims. Since the HIRA database covers nearly all of the nation's population, this database advantageously represents the entire Korean population, making it a valuable source for public health research.

\section{Study population and TRS}

This study was approved by the Institutional Review Board of Asan Medical Center (File Number: 2018-0131) and the requirement for informed consent from patients were exempted because the HIRA database consists of de-identified data.

We extracted the claim data from 1 January 2007 to 31 December 2016 (Serial number: M20180205894) from the HIRA database. This data included the following patient information: age, gender, date of admission, number of days of hospital admission, psychiatric diagnosis, prescribed medications. We then extracted data for patients who had at least one claim for the Korean Standard Classification of Diseases $(\mathrm{KCD})^{20}$ diagnostic code of F20 (schizophrenia) during the study period. As a reference, the KCD is based on the ICD-10. ${ }^{21}$

Using the data extracted for patients with schizophrenia, we defined an incident cohort to represent real-world situations more accurately. The incident cohort was defined as patients diagnosed with F20 for the first time between 2007 and 2016. The included patients had to have been antipsychotic-free for at least 1 year preceding the index point, to completely remove returning patients from the data-set, and patients had to have received antipsychotic treatment within 3 days of diagnosis. Patients with intellectual disability, pervasive developmental disorder and organic brain syndrome, for whom the schizophrenia diagnostic code is often used because of their psychotic symptoms, were also excluded. In addition, we further excluded patients who were under 12 or over 80 years of age at the time of diagnosis to maintain homogeneity of the included patients.

The cohort entry point was defined as the day when each patient was diagnosed with schizophrenia, and the follow-up time was from diagnosis to the end of the study period or death. TRS was defined as schizophrenia in which physicians prescribed more than two kinds of different antipsychotics for treatment. These antipsychotics have to be administered for at least 6 weeks and should reach the optimal therapeutic dosage of $10 \mathrm{mg}$ olanzapine or equivalent, after titration. Because this was a register-based study, we allowed some periods with polypharmacy.

\section{Analysis of before and after clozapine administration}

Several variables related to clinical courses were investigated and compared from before and after the use of clozapine. In particular, we compared the numbers of psychiatric admissions and emergency room visits, and the medication possession ratio (MPR). MPR, one of the most common measures of medication adherence, was calculated as the percentage of days of supply received divided by a period of time.

\section{Clozapine versus olanzapine}

We performed survival analysis to investigate the real-world effectiveness of clozapine longitudinally. To determine whether or not clozapine is superior in terms of real-world effectiveness, we compared clozapine with olanzapine for patients with TRS. As it is common to take multiple antipsychotics concurrently in many of these patients (pure monotherapy is relatively rare), we compared patients who used clozapine plus other antipsychotics other than olanzapine, versus patients who use olanzapine plus other antipsychotics other than clozapine in survival analysis. In addition, we also compared base demographics and clinical characteristics between these groups to further explain the differences in survival rates.

For survival analyses, both psychiatric admissions and emergency room visits were defined as the primary outcomes. The Kaplan-Meier survival curves were drawn for each survival analysis. To calculate hazard ratios for admissions and emergency room visits for clozapine compared with olanzapine, the Cox proportional hazards model as applied.

For sensitivity analysis, the age at disease onset and the number of antipsychotics used before clozapine or olanzapine were considered covariates to minimise selection bias.

\section{Current status of clozapine prescription}

Lastly, we investigated the current status of clozapine prescriptions. We estimated the mean treatment time before the initial use of clozapine, and also how many kinds of antipsychotics were used before clozapine. In so doing, we investigated the disparity between clinical guidelines and real-world practices. For the exclusion of unusually short or inappropriate administrations of antipsychotics, we set similar parameters as for investigating admissions. The antipsychotics that were administered for over 6 weeks and reached optimal therapeutic dosage after titration, as described in the Study population and TRS section above, were investigated.

\section{Statistical analyses}

We performed the paired $t$-test, Pearson chi-squared test and Kruskal-Wallis chi-squared test for the comparisons between patient groups. For survival analyses, we utilised the Cox proportional hazards model. All data were processed and analysed using R 3.4.1. ${ }^{22}$ Statistical significance was set at $P<0.05$.

\section{Results}

\section{Study population and demographics}

The number of patients who had at least one claim for the F20 (schizophrenia) diagnostic code totalled 448889 according to the HIRA data. This equated to $0.88 \%$ of the Korean national population, which was 51245707 in 2016.

The incident cohort was defined as patients diagnosed as F20 for the first time between 2007 and 2016 and consisted of 252299 patients. Within the incident cohort, 129341 patients were included after excluding intellectual disability, pervasive developmental disorder and organic brain syndrome. After excluding patients who were under 12 or over 80 years of age at the time of diagnosis, there were 126755 patients remaining. In total, 64442 patients were included in the final investigations, after excluding patients who did not receive antipsychotics within 3 days of diagnosis or who were not antipsychotic-free for 1 year preceding the index point.

The mean age of the incident cohort was 40.9 (s.d. $=15.6$ ) years, and 29335 patients were men. The patients in this cohort were treated for 1391.3 (s.d. $=1041.2$ ) days on average. In total, 52000 patients had experienced at least one treatment discontinuation, defined as a medication-free interval longer than 28 days. For the discontinued patients, an average of 2.29 (s.d. $=2.66$ ) discontinuations was reported. Figure 1 summarises patient eligibility and the flow of patients included in the study. 


\begin{tabular}{|c|c|c|c|c|c|}
\hline & $\begin{array}{c}\text { Clozapine } \\
(n=578)\end{array}$ & Olanzapine $(n=1470)$ & $\chi^{2}$ & d.f. & $P$ \\
\hline Male gender, $n$ & 259 & 646 & $0.093^{\mathrm{a}}$ & 1 & 0.7604 \\
\hline Onset age, years: mean (s.d.) & $30.5(12.9)$ & $36.6(14.1)$ & $82.007^{b}$ & 1 & $<0.001^{*}$ \\
\hline Time to start medication: mean (s.d.) & $1258.3(785.2)$ & $1268.9(748.7)$ & $0.444^{\mathrm{b}}$ & 1 & 0.505 \\
\hline Duration of treatment: mean (s.d.) & 407.5 (554.9) & 313.9 (438.4) & $5.431^{b}$ & 1 & 0.020 * \\
\hline
\end{tabular}

\section{Analysis of before and after clozapine administration}

In the incident cohort, 2338 patients have been prescribed clozapine. Of the 2338 patients who used clozapine, 998 patients were revealed as having TRS. The mean onset age was 33.5 (s.d. $=13.1$ ) years, and 465 patients $(46.6 \%)$ were men.

Among patients with TRS who used clozapine, the total number of psychiatric admissions was 3162 before clozapine introduction, and 1046 after. Moreover, the total number of emergency room visits, before and after clozapine introduction were 1067 and 254, respectively.

The total observation period before and after clozapine introduction was 1210842 days and 429571 days, respectively. Thus, the psychiatric admissions per unit day before and after clozapine introduction were 0.953 and 0.889 , respectively. Similarly, emergency room visits per unit day before and after clozapine introduction was 0.322 and 0.216 , respectively.

The average MPR was higher after clozapine introduction $(0.879$ (s.d. $=0.164)$ v. 0.767 (s.d. $=0.199), t=-14.5$, d.f. $=997$, $P<0.001)$. Table 1 shows a detailed comparison between before and after clozapine administration.

\section{Clozapine versus olanzapine}

There were 578 patients with TRS who used clozapine (who were also olanzapine-free) and 1470 patients who used olanzapine (who were also clozapine-free). The mean onset age for patients who used clozapine was 30.5 (s.d. $=12.9$ ) years, which was significantly younger than the 36.6 (s.d. $=14.1$ ) years of those who used olanzapine (Kruskal-Wallis $\chi^{2}=82.007$, d.f. $=1, P<0.001$ ).

The total duration of treatment was longer in the clozapine group than in the olanzapine group $(407.5$ (s.d. $=554.9)$ days $v$. 313.9 (s.d. $=438.4$ ) days, Kruskal-Wallis $\chi^{2}=5.431$, d.f. $=1, P=$ 0.020 ). Furthermore, it took an average of 1258.3 (s.d. $=785.2$ ) days on average to start clozapine, which was not statistically significantly different from the average of 1268.9 (s.d. $=748.7$ ) days for olanzapine (Kruskal-Wallis $\chi^{2}=0.444$, d.f. $=1, P=0.505$ ). Table 2 presents the demographics and clinical characteristics of the clozapine and olanzapine groups.

In survival analysis, olanzapine showed a superior survival rate for psychiatric admissions (hazard ratio $(\mathrm{HR})=0.615,95 \%$ CI
$0.523-0.722, P<0.001)$. There was no statistically significant difference in survival rates for emergency room visits between the clozapine and olanzapine groups ( $\mathrm{HR}=0.909,95 \% \mathrm{CI} 0.711-1.162, P=$ 0.447). Figure 2 shows the survival curves for clozapine versus olanzapine.

\section{Current status of clozapine and olanzapine use}

The mean treatment time before the initial usage of clozapine was 655.0 (s.d. $=512.6$ ) days. It was estimated that an average of 2.91 (s.d. $=1.16)$ kinds of antipsychotics were used before the prescription of clozapine. For olanzapine, the mean treatment time before the initial usage was 617.1 (s.d. $=524.8$ ) days. It was estimated that an average of 2.40 (s.d. $=0.72$ ) kinds of antipsychotics was used before the prescription of olanzapine. Table 3 show the current status of clozapine and olanzapine use.

\section{Discussion}

In this study, we tried to comprehensively investigate whether or not clozapine should always be used as the treatment of choice for patients with TRS. A few studies ${ }^{10-13}$ have suggested that clozapine may not be superior to other atypical antipsychotics such as olanzapine in terms of effectiveness. Indeed, in some cases, patients who were non-responsive to clozapine later respond to olanzapine. ${ }^{23}$

As a high-efficacy antipsychotic, olanzapine has been considered as superior to other atypical antipsychotics. ${ }^{14,24}$ Based on this perspective, and considering the risk of serious adverse effects and the cost of routine laboratory check-ups, clozapine should be used conservatively. Thus, a comprehensive study using real-world data was warranted. Herein, we utilised the HIRA data from Korea to document the real-world effectiveness and appropriateness of clozapine as the treatment of choice for TRS, taking another plausible choice of antipsychotic into consideration.

\section{Main findings and interpretation of our findings}

We compared the clinical characteristics of patients with schizophrenia before and after starting clozapine. The numbers of psychiatric admissions and emergency room visits per year were lower in 
Patients had at least one claim for the F20 (schizophrenia) diagnostic code $(n=448889)$

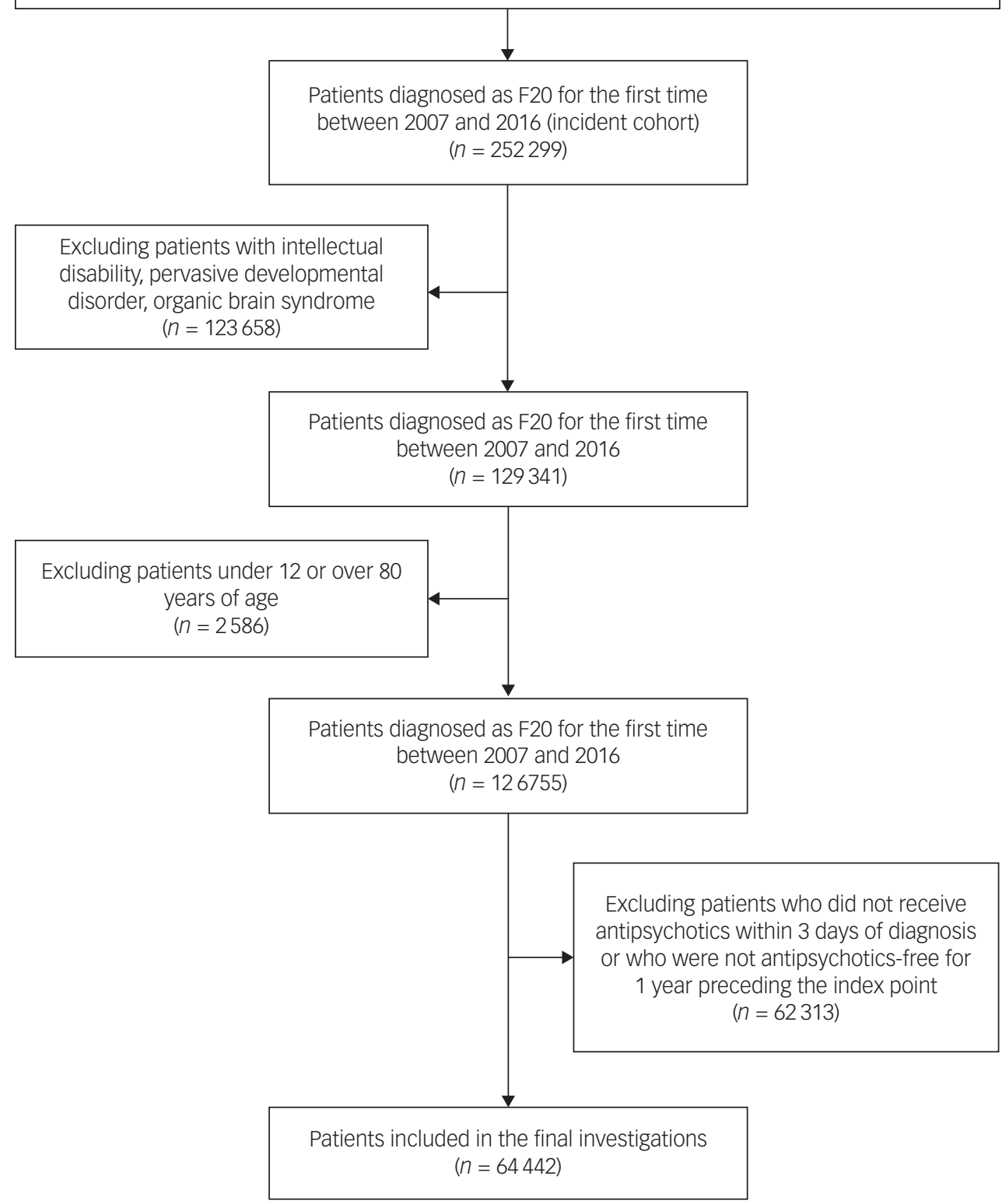

Fig. 1 Patient eligibility and the flow of patients included in or excluded from the study.

patients after starting clozapine, compared with before starting clozapine. However, due to the methodological limitations, we could not elucidate any statistical differences. We also identified that both admissions and emergency room visits rarely occurred. Furthermore, our study showed that MPR, a significant measure of treatment adherence, was higher after the introduction of clozapine. This suggests that treatment adherence for each patient may improve after the introduction of clozapine.

Despite a high MPR, clozapine did not show significant effectiveness superior to olanzapine in our primary survival analysis. In our survival analyses, the two different variables, psychiatric admissions and emergency room visits were examined and clozapine and olanzapine compared. These results were not supportive of clozapine, although the treatment adherence represented by MPR improved after clozapine initiation. In other words, clozapine did not show better clinical effectiveness despite better treatment adherence.

In the survival analysis, clozapine showed worse effectiveness in terms of admissions compared with olanzapine. As hospital admissions has been widely considered as an appropriate proxy for the clinical effectiveness of treatment, ${ }^{25-27}$ we could assume that olanzapine showed at least non-inferior effectiveness compared with clozapine within the patients with TRS data. In fact, some studies have already suggested that olanzapine may have at least the same effectiveness in patients with schizophrenia, especially in terms of cognitive effects, compared with clozapine. ${ }^{28-30}$ There were no statistically significant differences in the efficacies between clozapine and olanzapine in terms of positive or negative symptoms in a systematic review, although clozapine may have shown a little better efficacy than other antipsychotics. ${ }^{31}$ 
(a)

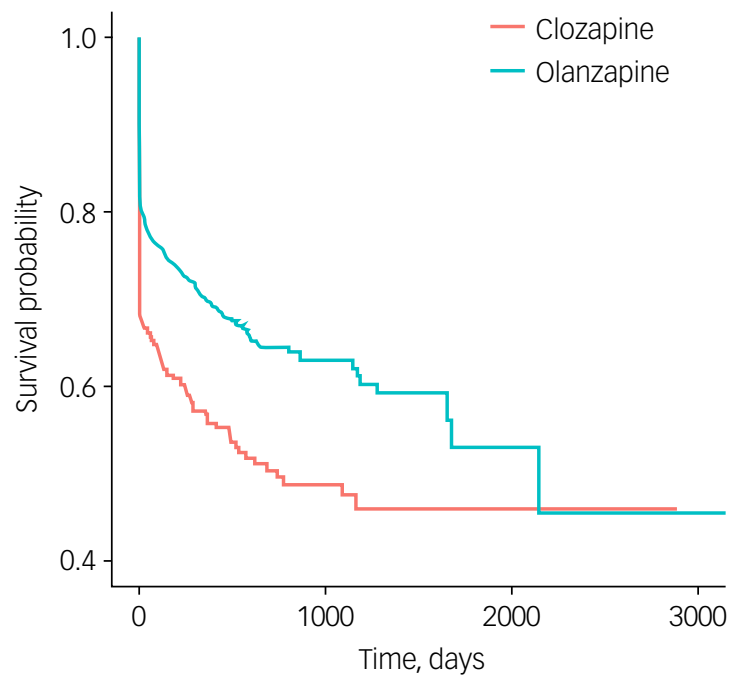

(b)

ER visits in patient with TRS

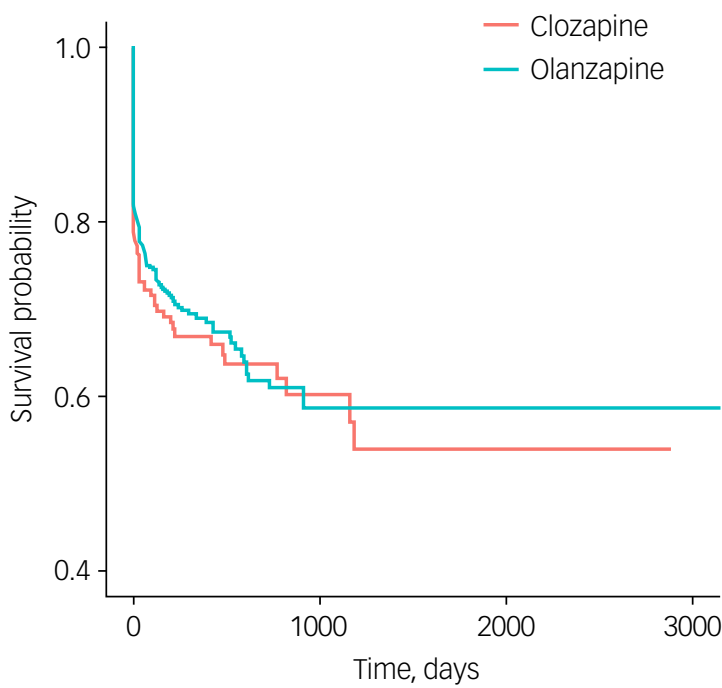

Fig. 2 Survival curves for clozapine versus olanzapine. (a) Psychiatric admissions to hospital and (b) emergency room (ER) visits by patients with treatment-resistant schizophrenia (TRS).

\section{Limitations}

There are some limitations to this study. Because our study is a register-based study, we could not completely rule out the possibilities of undetected earlier episodes in our investigated patients. Also, we could not entirely exclude returning patients since we set a 1year antipsychotic-free period to define the incident cohort. However, in a sensitivity analysis with a 3-year antipsychotic-free period, we found generally similar tendencies from the results. Moreover, there could be selection bias in the clozapine and olanzapine groups. As this was not a randomised controlled trial, patients who used clozapine could generally have more severe symptoms compared with patients who used olanzapine. Indeed, patients who used clozapine used more antipsychotics before the target antipsychotic (in this case, clozapine) and were also younger than those who used olanzapine in our demographic comparisons. It has been suggested that patients with early onset of schizophrenia show higher impulsivity. ${ }^{32}$ However, we achieved results with the same consistencies after introducing the age of disease onset and the number of antipsychotics used before clozapine or olanzapine in a sensitivity analysis. Furthermore, we tried to mitigate these limitations by restricting our focus of analysis within the patients with TRS who had already tried more than two different antipsychotics, which may have alleviate the possible differences in disease severity. Considering these limitations, we can only suggest that olanzapine is non-inferior to clozapine, with significantly better survival rates.

\begin{tabular}{|c|c|c|}
\hline & $\begin{array}{l}\text { Clozapine, } \\
\text { mean (s.d.) }\end{array}$ & $\begin{array}{l}\text { Olanzapine, } \\
\text { mean (s.d.) }\end{array}$ \\
\hline $\begin{array}{l}\text { Total treatment time before } \\
\text { using current medication, days }\end{array}$ & 898.9 (646.1) & $907.0(629.1)$ \\
\hline $\begin{array}{l}\text { Only those with adequate antipsychotic } \\
\text { treatment }{ }^{a}\end{array}$ & $655.0(512.6)$ & $617.1(524.8)$ \\
\hline $\begin{array}{l}\text { Number of different antipsychotics before } \\
\text { using medication }\end{array}$ & $5.75(1.86)$ & 4.49 (1.58) \\
\hline $\begin{array}{l}\text { Only those with adequate antipsychotic } \\
\text { treatment }\end{array}$ & 2.91 (1.16) & $2.40(0.72)$ \\
\hline
\end{tabular}

\section{Prescribing hesitancy}

Finally, we aimed to reveal the current status of clozapine use in this study, especially compared with current clinical guidelines. According to several guidelines relating to first-episode psychosis, the general recommendation is to use clozapine after two trial failures of antipsychotics with optimum dosages. ${ }^{3,33}$ For instance, it has been recommended to 'offer clozapine to people with schizophrenia whose illness has not responded adequately to treatment despite the sequential use of adequate doses of at least two different antipsychotic drugs' in the UK. ${ }^{3}$ Contrary to the clinical guidelines, we found that clinicians generally used clozapine far more conservatively. Some patients had been administered over nine different kinds of antipsychotics before clozapine was prescribed. In other words, clinicians tended to resist prescribing clozapine in real-world situations. A few studies have already identified this hesitancy among clinicians. ${ }^{34,35}$ This may in part be because of clozapine's dangerous adverse effects, such as agranulocytosis. However, taking our survival analysis into consideration, it may also be because of the recognised limited effectiveness of clozapine among clinicians in the real-world.

\section{Implications}

In conclusion, we investigated whether or not clozapine should always be considered the most effective antipsychotic in patients with TRS. We showed that olanzapine had higher survival rates than clozapine in terms of psychiatric admissions. Accordingly, it may be implied that olanzapine has at least non-inferior effectiveness compared with clozapine. This suggests that clozapine may not be the only treatment of choice for TRS. However, since many studies advocate the superior efficacy of clozapine, further longitudinal studies with extensive data spanning a few decades are recommended.

Young Tak Jo (D, MD, Department of Psychiatry, Asan Medical Center, University of Ulsan College of Medicine, Republic of Korea; Sung Woo Joo, MD, Department of

Psychiatry, Asan Medical Center, University of Ulsan College of Medicine, Republic of Korea; Soojin Ahn, MD, Department of Psychiatry, Asan Medical Center, University of Ulsan College of Medicine, Republic of Korea; Youngiae Choi, MD, Department of

Psychiatry, Asan Medical Center, University of Ulsan College of Medicine, Republic of Korea; Jungsun Lee, MD, PhD, Department of Psychiatry, Asan Medical Center, University of Ulsan College of Medicine, Republic of Korea

Correspondence: Jungsun Lee. Email: js_lee@amc.seoul.kr

First received 22 Sep 2020, final revision 1 Jun 2021, accepted 11 Jun 2021 


\section{Data availability}

The data that support the findings of this study are not publicly available because of the restrictions by the Health Insurance Review \& Assessment Service.

\section{Author contributions}

Y.T.J. contributed to the conceptualisation, data curation, formal analysis, methodology, writing - original draft. S.W.J. contributed to the conceptualisation, methodology, writing - review and editing. S.A. contributed to the methodology, writing - review and editing. Y.C. contributed to the writing - review and editing. J.L. contributed to the conceptualisation, project administration, supervision, data curation, formal analysis, methodology, writing - review and editing.

\section{Declaration of interest}

The authors have no conflicts of interest to disclose.

\section{References}

1 Lambert M, Karow A, Leucht S, Schimmelmann BG, Naber D. Remission in schizophrenia: validity, frequency, predictors, and patients' perspective 5 years later. Dialogues Clin Neurosci 2010; 12: 393-407.

2 Elkis H, Buckley PF. Treatment-resistant schizophrenia. Psychiatr Clin North Am 2016; 39: 239-65.

3 National Institute for Health and Care Excellence. Psychosis and Schizophrenia in Adults: Treatment and Management: Updated Edition 2014. National Collaborating Centre for Mental Health, 2014.

4 American Psychiatric Association. The American Psychiatric Association Practice Guideline for the Treatment of Patients with Schizophrenia. American Psychiatric Association, 2020.

5 Wiciński M, Weclewicz MM. Clozapine-induced agranulocytosis/granulocytopenia: mechanisms and monitoring. Curr Opin Hematol 2018; 25: 22-8.

6 Kane JM. Clinical efficacy of clozapine in treatment-refractory schizophrenia: an overview. Br J Psychiatry Suppl. 1992; 160 (Suppl 17): 41-5.

7 Cho J, Hayes RD, Jewell A, Kadra G, Shetty H, MacCabe JH, et al. Clozapine and all-cause mortality in treatment-resistant schizophrenia: a historical cohort study. Acta Psychiatr Scand 2019; 139: 237-47.

8 Wimberley T, MacCabe JH, Laursen TM, Sørensen HJ, Astrup A, Horsdal HT, et al. Mortality and self-harm in association with clozapine in treatment-resistant schizophrenia. Am J Psychiatry 2017; 174: 990-8.

9 Jin H, Tappenden P, MacCabe JH, Robinson S, Byford S. Evaluation of the costeffectiveness of services for schizophrenia in the UK Across the entire care pathway in a single whole-disease model. JAMA Netw Open 2020; 3 : e205888-e.

10 Naber D, Riedel M, Klimke A, Vorbach EU, Lambert M, Kühn KU, et al. Randomized double blind comparison of olanzapine vs. clozapine on subjective well-being and clinical outcome in patients with schizophrenia. Acta Psychiatr Scand 2005; 111: 106-15.

11 Tollefson GD, Birkett MA, Kiesler GM, Wood AJ. Double-blind comparison of olanzapine versus clozapine in schizophrenic patients clinically eligible for treatment with clozapine. Biol Psychiatry 2001; 49: 52-63.

12 Bitter I, Dossenbach MR, Brook S, Feldman PD, Metcalfe S, Gagiano CA, et al. Olanzapine versus clozapine in treatment-resistant or treatment-intolerant schizophrenia. Prog Neuropsychopharmacol Biol Psychiatry 2004; 28: 173-80.

13 Samara MT, Dold M, Gianatsi M, Nikolakopoulou A, Helfer B, Salanti G, et al. Efficacy, acceptability, and tolerability of antipsychotics in treatment-resistan schizophrenia: a network meta-analysis. JAMA Psychiatry 2016; 73: 199-210.

14 Komossa K, Rummel-Kluge C, Hunger H, Schmid F, Schwarz S, Duggan L, et al Olanzapine versus other atypical antipsychotics for schizophrenia. Cochrane Database Syst Rev 2010; 3: Cd006654.

15 Stanworth D, Hunt NC, Flanagan RJ. Clozapine-a dangerous drug in a clozapinenaïve subject. Forensic Sci Int 2012; 214: e23-5.
16 Tiihonen J, Mittendorfer-Rutz E, Majak M, Mehtälä J, Hoti F, Jedenius E, et al. Real-world effectiveness of antipsychotic treatments in a nationwide cohort of 29823 patients with schizophrenia. JAMA Psychiatry 2017; 74: 686-93.

17 Bitter I, Katona L, Zámbori J, Takács P, Fehér L, Diels J, et al. Comparative effectiveness of depot and oral second generation antipsychotic drugs in schizophrenia: a nationwide study in Hungary. Eur Neuropsychopharmacol 2013; 23 : 1383-90.

18 Taipale $\mathrm{H}$, Mehtälä J, Tanskanen A, Tiihonen J. Comparative effectiveness of antipsychotic drugs for rehospitalization in schizophrenia-a nationwide study with 20-year follow-up. Schizophr Bull 2018; 44: 1381-7.

19 Kim JA, Yoon S, Kim LY, Kim DS. Towards actualizing the value potential of korea health insurance review and assessment (HIRA) data as a resource for health research: strengths, limitations, applications, and strategies for optimal use of HIRA data. J Korean Med Sci 2017; 32: 718-28.

20 Korea S. Korean Standard Classification of Diseases. Seoul: Statistics Korea, 2010.

21 World Health Organization. The ICD-10 classification of mental and behavioural disorders: diagnostic criteria for research. World Health Organization, 1993.

22 R Core Team. R: A language and environment for statistical computing. R Core Team, 2013.

23 Dossenbach MRK, Beuzen J-N, Avnon M, Belmaker RH, Elizur A, Mark M, et al. The effectiveness of olanzapine in treatment-refractory schizophrenia when patients are nonresponsive to or unable to tolerate clozapine. Clin Ther 2000; 22: $1021-34$

24 Citrome L, McEvoy JP, Todtenkopf MS, McDonnell D, Weiden PJ. A commentary on the efficacy of olanzapine for the treatment of schizophrenia: the past, present, and future. Neuropsychiatr Dis Treat 2019; 15: 2559-69.

25 Burns T. Evolution of outcome measures in schizophrenia. Br J Psychiatry 2007; 191 (Suppl S50): s1-6.

26 Burns T. Hospitalisation as an outcome measure in schizophrenia. $\mathrm{Br} J$ Psychiatry 2007; 191 (Suppl S50): s37-41.

27 Addington $D E$, McKenzie $E$, wang J. Validity of hospital admission as an outcome measure of services for first-episode psychosis. Psychiatr Serv 2012; 63: $280-2$.

28 Bilder RM, Goldman RS, Volavka J, Czobor P, Hoptman M, Sheitman B, et al. Neurocognitive effects of clozapine, olanzapine, risperidone, and haloperidol in patients with chronic schizophrenia or schizoaffective disorder. Am J Psychiatry 2002; 159: 1018-28.

29 Sharma T, Hughes C, Soni W, Kumari V. Cognitive effects of olanzapine and clozapine treatment in chronic schizophrenia. Psychopharmacology 2003; 169: 398-403.

30 Nielsen RE, Levander S, Kjaersdam Telléus G, Jensen SOW, Østergaard Christensen T, Leucht S. Second-generation antipsychotic effect on cognition in patients with schizophrenia-a meta-analysis of randomized clinical trials. Acta Psychiatr Scand 2015; 131: 185-96.

31 Lobos CA, Komossa K, Rummel-Kluge C, Hunger H, Schmid F, Schwarz S, et al. Clozapine versus other atypical antipsychotics for schizophrenia. Cochrane Database Syst Rev 2010; 11: Cd006633.

32 Immonen J, Jääskeläinen $\mathrm{E}$, Korpela $\mathrm{H}$, Miettunen J. Age at onset and the outcomes of schizophrenia: a systematic review and meta-analysis. Early Interv Psychiatry 2017; 11: 453-60.

33 Lehman AF, Lieberman JA, Dixon LB, McGlashan TH, Miller AL, Perkins DO, et al. Practice guideline for the treatment of patients with schizophrenia, second edition. Am J Psychiatry 2004; 161 (2 Suppl): 1-56.

34 Gee S, Vergunst F, Howes $O$, Taylor D. Practitioner attitudes to clozapine initiation. Acta Psychiatr Scand 2014; 130: 16-24.

35 Warnez S, Alessi-Severini S. Clozapine: a review of clinical practice guidelines and prescribing trends. BMC Psychiatry 2014; 14: 102. 